\title{
Mgll linear polarization measurements using the MSFC Solar Ultraviolet Magnetograph
}

\author{
Edward West ${ }^{\text {a }}$, Jonathan Cirtain ${ }^{\text {a }}$, Ken Kobayashi ${ }^{\text {b }}$, John Davis ${ }^{\mathrm{b}}$, \\ Allen Gary ${ }^{b}$ and Mitzi Adams ${ }^{a}$ \\ ${ }^{a}$ Marshall Space Flight Center/NASA, 320 Sparkman Dr., Huntsville, AL 35805-1912 \\ ${ }^{\mathrm{b}}$ The University of Alabama in Huntsville, 320 Sparkman Dr., Huntsville, AL 35805-1912
}

\begin{abstract}
This paper will describe the Marshall Space Flight Center's Solar Ultraviolet Magnetograph (SUMI) sounding rocket program, with emphasis on the polarization characteristics of the VUV optics and their spectral, spatial and polarization resolution. SUMI's first flight (7/30/2010) met all of its mission success criteria and this paper will describe the data that was acquired with emphasis on the MgII linear polarization measurements.
\end{abstract}

Keywords: MgII polarization, VUV, SUMI, Solar Ultraviolet Magnetograph, VUV spectrograph, sounding rocket.

\section{INTRODUCTION}

This section will describe the science goals $(\S 1.1)$ of the Marshall Space Flight Center (MSFC) Solar Ultraviolet Magnetograph (SUMI), describe the instrument with emphasis on the spectrograph and polarimeter $(\$ 1.2)$ and summarize the observations were made during SUMI's first flight.

\subsection{Science goals}

SUMI's goal is to measure the magnetic field in the upper chromosphere/lower transition region of the Sun's atmosphere. The reason to make those measurements is given in the following paragraphs:

The problem of how energy, stored in the solar magnetic field, is released to heat the corona and drive the dynamic phenomena of the outer atmosphere, flares and coronal mass ejections, remains unsolved. Neither theoretical or numerical models nor current observations are able to provide a conclusive explanation. A substantial fraction of this energy propagates outward into the interplanetary medium in the form of electromagnetic (EM) radiation, energetic particles, and the solar wind that creates, controls and modifies the structure of the interplanetary medium. Currently space weather effects orbiting satellites, and in the future, these will be a major concern for astronauts working on the lunar surface and traveling through interplanetary space. The solar atmosphere dynamics are the initial conditions for any model that describes and predicts space weather. Therefore understanding the processes that contribute to both the steady and transient release of magnetic energy into the solar atmosphere is a crucial element of these studies.

To understand the fundamental processes that lead to the explosive release of magnetic energy in solar flares and that initiates and propels coronal mass ejections (CMEs), the magnetic field needs to be observed and its topology and evolution inferred prior to and during the energy release process . Success has been achieved in the characterization of the full vector magnetic field in the photosphere, where $\beta$, the ratio of the gas pressure to the magnetic pressure, is $\geq 1$. At higher levels in the atmosphere (Figure 1$)^{1}$ where $\beta<1$, the magnetic field, through the Lorentz force, controls the structure and dynamics of the solar plasma, and rapid changes in its structure can produce the observed energetic

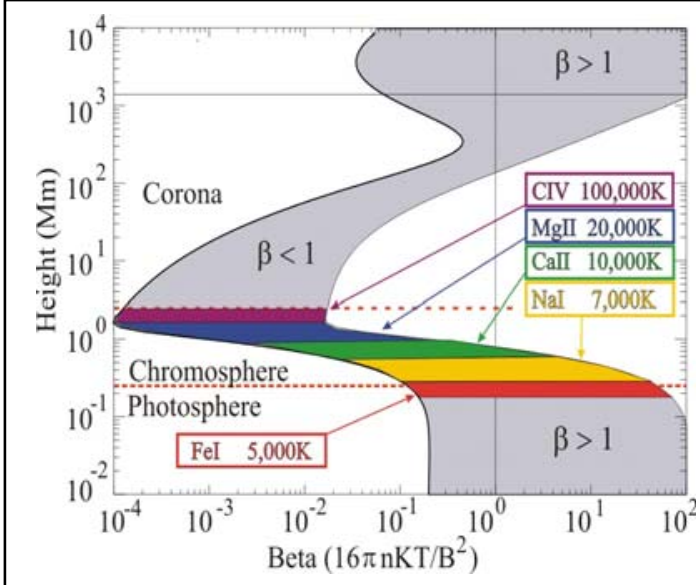

Figure 1. Ratio of gas pressure to magnetic pressure $(\beta)$ as a function of height, for an assumed range of photospheric magnetic field strength of 100 to $2500 \mathrm{G}^{1}$. 
events. However, observations of the magnetic field at these higher levels have proven to be difficult, placing a serious limitation on our understanding of the physical processes occurring there.

The missing element is the ability to understand and describe quantitatively the transition layer where the field is no longer pressure-dominated as in the photosphere. The need to understand the energy release processes was identified two decades $\mathrm{ago}^{2}$, and was reaffirmed as the major conclusion of a workshop on the definition of the scientific goals for "Beyond Solar-B" ${ }^{3}$. To understand this process, full vector magnetic field measurements at several different heights in the solar atmosphere, from the photosphere to the upper transition region where the field becomes nearly force free, will be required. The vector field in the photosphere and low chromosphere has been measured with ground-based instruments. Space-based instruments such as the Michelson Doppler Interferometer (MDI) on the Solar \& Heliospheric Observatory (SoHO) have demonstrated that continuous, distortion free, observations of the longitudinal magnetic field from space are very important in our understanding of the Sun's magnetic field. The Solar Optical Telescope (SOT) on Hinode and the Helioseismic and Magnetic Imager (HMI) on the Solar Dynamics Observatory (SDO) spacecraft are now extending our space based observations to include photospheric vector magnetic fields. Although these high resolution observations will greatly advance our knowledge of the behavior of the magnetic field in the photosphere, they shed little information on how the magnetic field higher in the solar atmosphere transitions to a nearly force-free state. This requires measurement of the field using spectral lines that are formed at temperatures above $20,000{ }^{\circ} \mathrm{K}$. The two spectral lines chosen for SUMI are strong resonance pairs of MgII at $280 \mathrm{~nm}$ and CIV at $155 \mathrm{~nm}$. Because these lines occur in the vacuum ultraviolet (VUV), they are inaccessible from the ground. Together with the FeI, NaI and CaII lines, they form a quintet of strong magnetically sensitive lines which can be used in future missions to spatially map the magnetic field in three dimensions. Such a future space mission is the Magnetic Transition Region Probe (MTRAP) ${ }^{4}$. One of the objectives for the Solar Ultraviolet Magnetograph Investigation (SUMI) is the demonstration that the MgII and CIV lines can meet the requirements for this force-free region.

Goal for the SUMI sounding rocket program: To make vector magnetic field measurements in the upper chromosphere and lower transition region.

The Ultraviolet Spectrometer and Polarimeter (UVSP) instrument on board the Solar Maximum Mission (SMM, 1980) was the first space-based instrument to try to map and understand polarization in the transition region. The UVSP observations of MgII suggested that this line was a potential candidate for magnetic field measurements in the upper chromosphere ${ }^{5}$. Although UVSP attempted measurements of the CIV line, poor photon statistics and the loss of the ability to observe the CIV lines early in the mission resulted in a failure to produce convincing evidence that longitudinal magnetic field measurements were possible. While the scientific value of these observations has been acknowledged $^{6,7}$, the measurements are difficult because they are in the vacuum ultraviolet (VUV) wavelength range where the optical efficiency of standard optics is low. The SUMI sounding rocket program was born from several development programs ${ }^{8,9,10}$ to improve the overall wavelength and polarization efficiency for the CIV (155nm) measurement while including the MgII measurements in its observing program. While these development programs were successful, accommodating the dual-line instrument in a sounding rocket envelope has been challenging.

Measuring CIV has always been the driving force in the development of SUMI. This resonance line is formed in the relatively thin transition region which simplifies its interpretation, although the actual height of formation can be problematic. However, the most difficult instrumental problem is the weak line splitting in UV. As a result the magnetic sensitivity, $\lambda^{2} \mathrm{~g}$, where $\mathrm{g}$ is the Landé $\mathrm{g}$ factor and $\lambda$ is the wavelength, is low compared to lines in the visible and infrared. Table 1 compares the SUMI lines with lines that are being used in photospheric and low chromospheric magnetographs. Certainly the results from SUMI will impact future missions as scientist try to

Table 1: This table shows a comparison of candidate lines that are being developed for current and future space-based photospheric, chromospheric and transition region vector magnetic field measurements.

\begin{tabular}{|c|c|c|c|c|c|}
\hline $\begin{array}{c}\text { Spectral } \\
\text { lines }\end{array}$ & $\begin{array}{c}\lambda \\
(\mathrm{nm})\end{array}$ & $\mathrm{g}$ & $\begin{array}{c}\lambda^{2} \mathrm{~g} \\
\mathrm{~mm}^{2}\end{array}$ & $\begin{array}{c}\text { Height } \\
(\mathrm{km})\end{array}$ & Remarks \\
\hline \multicolumn{8}{|c|}{ Chromosphere } \\
\hline CIV & $\mathbf{1 5 5 . 0}$ & $\mathbf{1 . 2}$ & $\mathbf{0 . 2 9}$ & $\mathbf{2 2 0 0}$ & SUMI \\
\hline MgII & $\mathbf{2 8 0 . 0}$ & $\mathbf{1 . 2}$ & $\mathbf{0 . 9 4}$ & $\mathbf{2 0 0 0}$ & SUMI \\
\hline CaII & $\mathbf{8 5 4 . 2}$ & $\mathbf{1 . 1}$ & $\mathbf{8 . 0 2}$ & $\mathbf{1 3 1 9}$ & \\
\hline NaI & $\mathbf{5 8 9 . 5}$ & 1.3 & 4.51 & $\mathbf{7 0 0}$ & \\
\hline \multicolumn{7}{|c|}{ Photosphere } \\
\hline FeI & $\mathbf{6 3 0 . 2}$ & $\mathbf{2 . 5}$ & $\mathbf{9 . 9 2}$ & $\mathbf{2 5 0}$ & Hinode \\
\hline
\end{tabular}

MTRAP $^{6}$ : The Magnetic Transition Region Probe has all of these lines in its baseline mission requirements. 


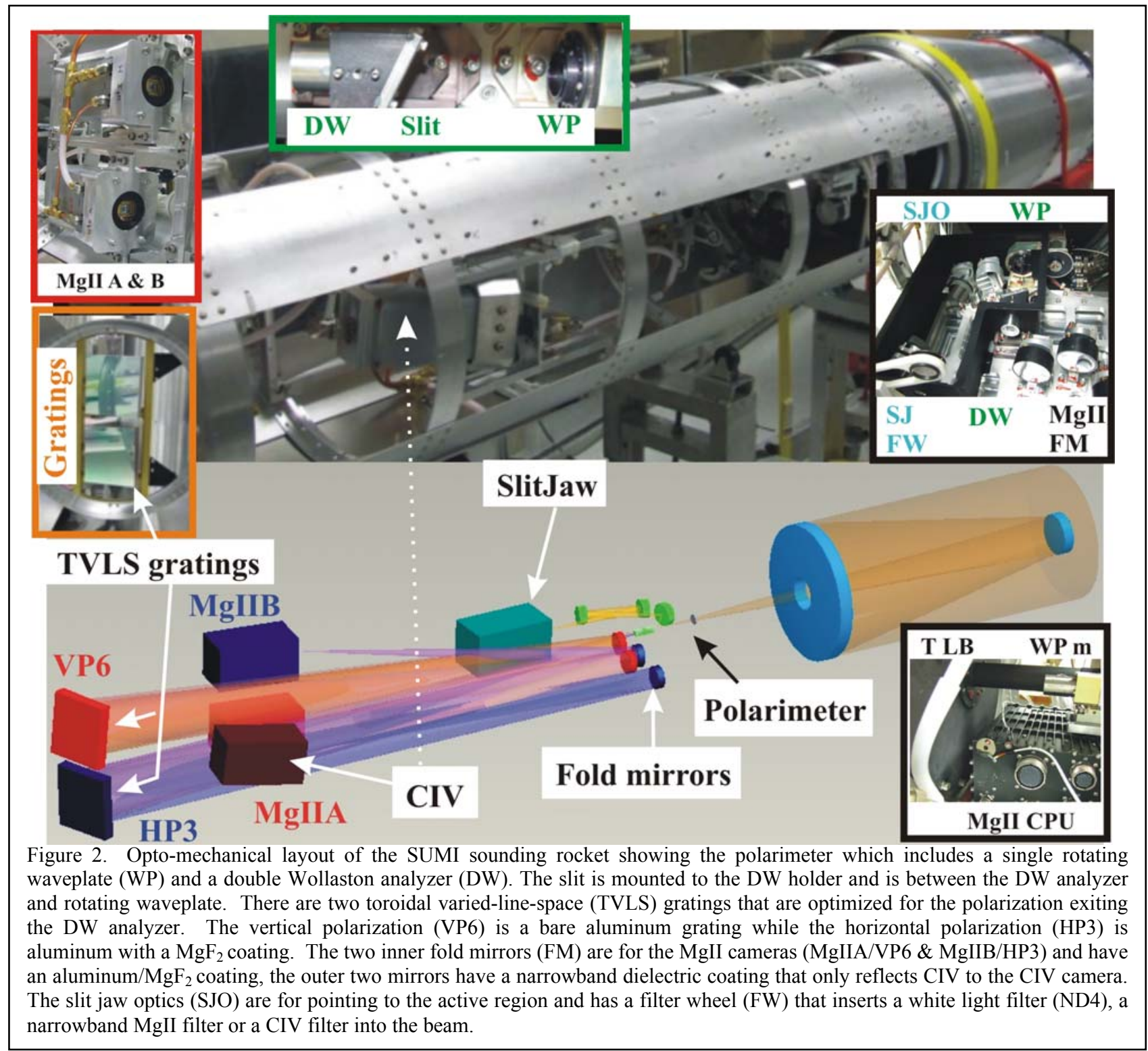

understand the 3-D structure of the Sun's magnetic field.

\subsection{Instrument description}

The instrument package includes a spectro-polarimeter for the scientific observations and a slitjaw camera system for pointing (white light images) and two "narrowband" filters to provide context images of the MgII (FWHM 40nm) and CIV (FWHM 30nm) lines. SUMI has been described in detail in several SPIE papers ${ }^{10,12}$.

In Figure 2 the optical model of SUMI is shown along with the mechanical components that determine the spectral and polarization resolution of the instrument. The SUMI telescope is an innovative "cold mirror" design that achieves its spectral filtering through the use of multilayer coatings. An important design requirement for solar telescopes is the rejection of the unwanted solar energy before it is concentrated onto the secondary. The standard solution is a Gregorian design that places the primary focus onto a field stop in front of the secondary mirror that rejects most of the energy at the expense of a reduced field of view. Gregorian telescopes are physically longer than Cassegrain or Ritchey-Chretien designs where the secondary mirror is placed between the primary and the telescope focus. Because our measurements are only interested in two narrow wavebands, we can use a "cold mirror" design to reject the unwanted energy. Customized dielectric high reflectivity (DHR) coatings are applied to both the primary and secondary. The dielectric 
coating reflects only the two spectral bands covering the CIV and MgII wavelengths. The measured out-of-band rejection of the coatings remains excellent across the optical and infrared wavelengths. The coatings for the MgII lines $(280 \mathrm{~nm})$ are deposited first. This coating was adjusted to minimize the reflectivity at $310 \mathrm{~nm}$ that would contaminate the observations of the $2^{\text {nd }}$ order CIV $(155 \mathrm{~nm})$ measurement. The CIV dielectric stack is then deposited on top of the MgII coating. This minimizes the absorption loss at CIV. The unwanted visible and infrared wavelengths incident on the primary mirror pass through the DHR coatings and fused silica mirror where they strike a spherically figured, aluminum coated back surface. This back surface reflects this incident radiation as a collimated beam out through the entrance aperture of the telescope into space. The front surface of the secondary has the same dielectric coatings to further reduce the out-of-band wavelengths ( $4 \%$ from primary mirror). To reduce the out-of-band light further, the back of the secondary mirror has an anti-reflection coating followed by a light-absorbing cone ${ }^{10}$.

Our Ritchey-Chretien design provides a compact telescope which, within a fixed instrument length, increases the spectrograph length and, therefore, the spectral resolution. This design is an on-axis, radially symmetric, telescope that minimizes instrumental polarization.

\subsubsection{Polarimeter Design}

The polarimeter consists of a rotating waveplate located in front of the spectrograph slit with a double Wollaston analyzer mounted behind the slit. Because of the low flux and weak linear polarization signal of the CIV emission line, our plan was to measure the circular polarization (longitudinal field) at CIV with exploratory linear polarization

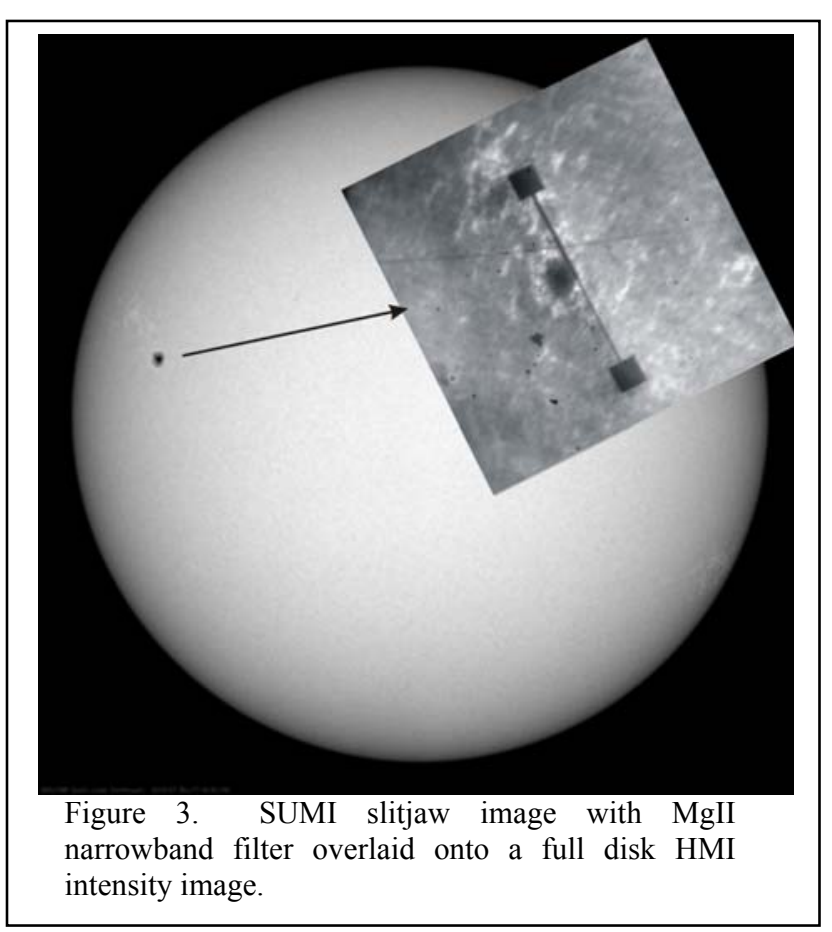
measurements to verify our radiometry calculations. For MgII, whose intensity is two orders of magnitude larger than CIV, both linear and circular polarization measurements are possible.

The waveplate is a zero order $\mathrm{MgF}_{2}$ design that is a "quarterwave" retarder at $\mathrm{CIV}\left(\sim 283^{\circ}\right)$ and a $\sim 138^{\circ}$ retarder at $\mathrm{MgII}^{12}$. The strength of the MgII line allows approximately one hundred polarization images to be acquired in the same time as a single CIV polarization map. Therefore, the circular polarization efficiency of the SUMI waveplate is maximized at $\sim 100 \%$ at CIV while still achieving $\sim 70 \%$ at $\mathrm{MgII}$. While the polarization efficiency does not affect the exposure time, which is dictated by photon statistics; it does affect the time required to obtain a given polarization resolution.

The SUMI analyzer is a double Wollaston polarizer that uses the birefringence of $\mathrm{MgF}_{2}$ to split the incident light into two linear polarization signals, thereby achieving both high broadband polarization efficiency and a total transmission greater than $50 \%$, even at $\mathrm{CIV}^{9}$.

\subsubsection{Spectrograph Design}

The SUMI spectrograph consists of two toroidal variedline-space (TVLS) gratings ${ }^{13,14}$, one for each linear polarization channel exiting the double Wollaston analyzer. Toroidal gratings were used to minimize the aberrations associated with the double Wollaston analyzer.

To optimize the instrument's imaging performance within a compact envelope, the spectrograph was designed to measure CIV in $2^{\text {nd }}$ order and MgII in $1^{\text {st }}$ order. The two TVLS gratings each have the same ruling variations as a function of position across their surface. This ruling variation differs by about $2 \%$ around the central groove density of 2440 lines per $\mathrm{mm}$. Both gratings have the same concave toroidal radii of curvature: $1500 \mathrm{~mm}$ in the dispersion axis and $1602 \mathrm{~mm}$ in the spatial direction. With this design, the spectrograph's wavelength resolution is significantly improved while minimizing reflection losses by avoiding additional optics that would be required by other spectrograph designs ${ }^{13}$. 


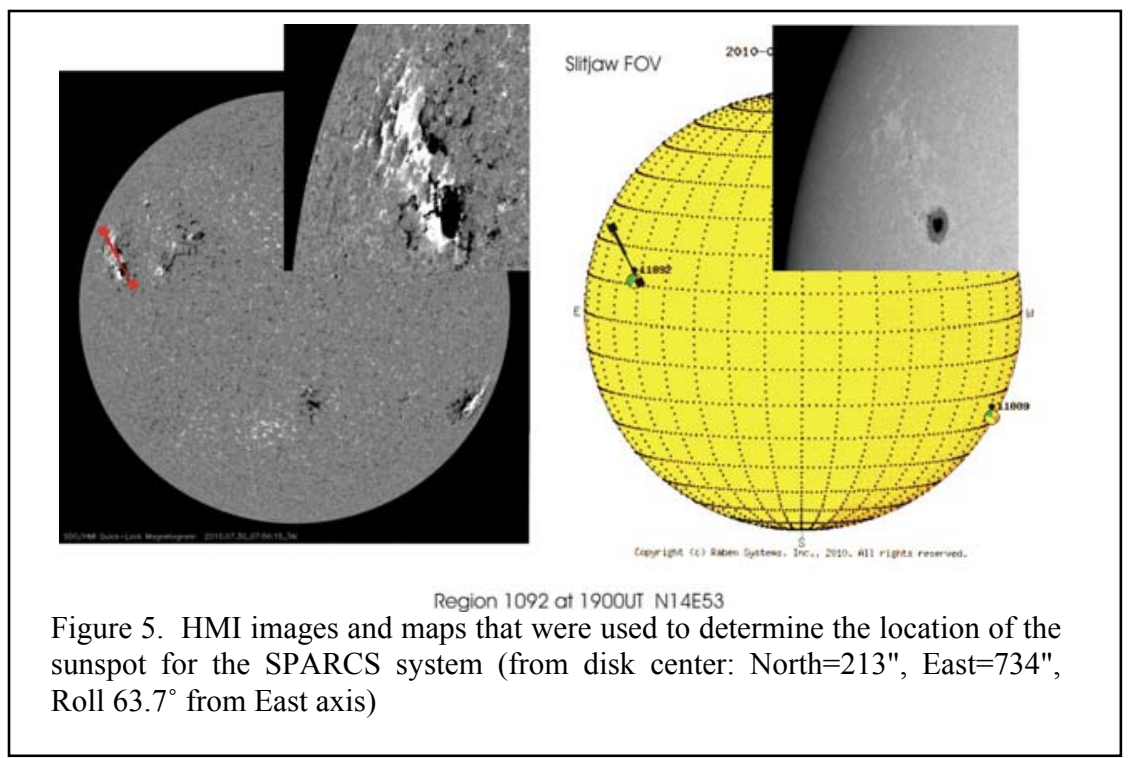

Following the gratings, fold mirrors redirect the CIV and MgII polarization states onto their own wavelength specific camera. The two MgII fold mirrors are aluminum, while the CIV mirrors have the same CIV DHR coatings that are used on the telescope mirrors ${ }^{10}$. This reflection is an additional filter to reduce the $310 \mathrm{~nm}$ contamination of the CIV line.

\subsubsection{The Slit and Slit-Jaw Camera}

SUMI's slit has a unique structure for our exploratory observations. The slit is cut into a $6 \mathrm{~mm}$ thick diamond turned aluminum substrate and is $\sim 3.7 \mathrm{~mm}$ long ( 3.5 arc minutes) with $0.5 \times 0.5 \mathrm{~mm}(30 \mathrm{arcsec})$ boxes on each end. The width of the slit has been split into two sections, a $30 \mu \mathrm{m}(\sim 1.6$ arc seconds) and a $60 \mu \mathrm{m}(\sim 3.2 \mathrm{arc}$ second). Due to the exploratory nature of the SUMI measurements, the uncertainty in the target at launch and the limited observing time of the flight ( $\sim 6$ minutes), this structure was adopted to give SUMI a larger dynamic range and to capture enough photons to make meaningful polarization measurements at CIV.

The slit-jaw camera has three functions: 1) to enable the operator to place the spectrograph slit on the region of interest during target acquisition; 2) to record any jitter or drift in the pointing system during the flight; and 3) to provide context images for the grating scans after the target is acquired. This camera has a 5.6 ${ }^{\prime}$ x 5.6 field of view. To improve the readout rate during target acquisition, the camera uses $2 \times 2$ on chip binning. The camera changes back into full resolution (binning 1x1) after the target is acquired.

\subsubsection{Spectrograph cameras}

The MgII, CIV and slitjaw cameras have the same optical characteristics. These E2V CCDs use a bare silicon structure (BN) to enhance their VUV sensitivity. When these cameras were ordered, the BN processing was only available in the $512 \times 512 \times 13 \mu \mathrm{m}$ pixel frame transfer format and a $1024 \times 256 \times 26 \mu \mathrm{m}$ full frame format. The $13 \mu \mathrm{m}$ pixel size corresponds to a plate scale for the MgII cameras of $20.3 \mathrm{~m} \AA$ and $0.46 \mathrm{arcsec}$ per pixel (twice the TVLS resolution). The CIV camera ( $26 \mu \mathrm{m}$ pixels) corresponds to a plate scale of $18.8 \mathrm{~m} \AA$ and 0.91 arcsec per pixel.

\subsection{Flight characteristics}

Even though the solar cycle was just coming out of solar minimum in the spring of 2010, we were fortunate that SUMI was launch (7/30/2010) just as Active Region 11092 (AR) emerged and rotated onto the disk. This region was a simple $\mathrm{H}$-type sunspot that was an ideal candidate for relating the magnetic field measured in the photosphere to the magnetic field in the upper chromosphere/lower transition region. However, the location of the AR did not meet our original launch criteria (within 0.5 solar radius of disk center). With no launch window available the following week and a launch delay of at least two weeks (and no guarantee of a sunspot), the decision was made to continue with the launch. While 
converting SUMI's polarization measurements into magnetic fields would have been difficult due to the "large" optical depth of the CIV and MgII lines, this region would provide stronger linear polarization signals. Therefore, SUMI's MgII measurements, which measures the full Stokes vector, would benefit from these stronger linear polarization signals.

Our CIV radiometry calculations showed that linear polarization measurements were not possible for a sounding rocket's 6 minute observing time. While the location of the AR might help the MgII linear measurements, the strength of the CIV circular polarization signals would be weaker. Therefore, the location of the active region was less than optimum for CIV but was more than adequate for evaluating the engineering and technology performance of SUMI.

\section{DESCRIPTION OF OBSERVATIONS}

Because of programmatic requirements, SUMI was prepared for launch during the summer of 2008. Since SUMI requires an active region to make its magnetic field/polarization measurements that launch date slipped to 2009 and finally to July 30, 2010 due to the Sun's extended solar minimum. This initial flight was a full engineering flight: (1.) to verify that the cold mirror technology on SUMI's telescope mirrors could be used as a "prefilter" to isolate the wavelength bands for future solar telescope designs, (2.) to show that simultaneous spectro-polarimetry measurements of orthogonal polarizations could be made on the two CIV emission lines (155.0 and 154.8nm) and the two MgII emission lines $(279.8$ and $280.4 \mathrm{~nm})$, (3.) to verify that the mechanical structure and fixtures could maintain the tight optical alignment tolerances, (4.) to show that the commercially-acquired cameras could survive a $10 \mathrm{G}$ launch, and (5.) to obtain images to verify and adjust the radiometry calculations and optimize the exposure times for future flights.

While the mission met all of its major engineering success criteria, there were two unexpected problems that limited the science for SUMI. This section will describe how the performance of the waveplate (\$2.1) allowed only MgII linear polarization measurements and how the pointing system $(\S 2.2)$ reduced the polarization resolution by introducing image motion in our polarization measurements.

\subsection{Waveplate failure}

Table 2 shows the type of measurements that SUMI was suppose to make during its flight. At altitudes above 200km, the mission concentrates on CIV circular polarization [V] measurements with the waveplate fast axis rotating between $45^{\circ} / 225^{\circ}$ and $135^{\circ} / 315^{\circ}$ positions (red in Table2). Under $200 \mathrm{~km}$ where the CIV lines are weak, SUMI concentrates on the MgII linear polarization measurements $[\boldsymbol{Q} \& \boldsymbol{U}]$. With that in mind, the waveplate fast axis position (FA) at launch is at $0^{\circ}$.

Table 2. Polarization Response to SUMI's rotating waveplate $\left(\Delta=\right.$ measured retardance $\left.{ }^{11}\right)$. With the waveplate rotation failure, the only measurements that were made were with the fast axis (FA) at $0^{\circ}$. Note: the waveplate FA at $45^{\circ}$ is equivalent to $\mathrm{FA}$ at $225^{\circ}$.

\begin{tabular}{|c|c|c|c|c|}
\hline Waveplate & \multicolumn{2}{|c|}{$\begin{array}{c}\text { CIV } \\
\Delta=282.9^{\circ}\end{array}$} & $\begin{array}{c}\text { MgII } \\
\Delta=138.2^{\circ}\end{array}$ \\
\hline FA & $2 * \mathrm{VP}$ & $2 * \mathrm{HP}$ & $2^{* \mathrm{HP}}$ \\
\hline $45^{\circ}$ & $I+0.22 Q+97 \mathrm{~V}$ & $I-0.22 Q-97 \mathrm{~V}$ & $I-0.75 Q-0.67 \mathrm{~V}$ & $I+0.75 Q+0.67 \mathrm{~V}$ \\
\hline $135^{\circ}$ & $I+0.22 Q-97 \mathrm{~V}$ & $I-0.22 Q+97 \mathrm{~V}$ & $I-0.75 Q+0.67 \mathrm{~V}$ & $I+0.75 Q-0.67 V$ \\
\hline $0^{\circ}$ & $I+Q$ & $I-Q$ & $I+Q$ & $I-Q$ \\
\hline $22.5^{\circ}$ & $I+.63 Q+.39 U+.68 \mathrm{~V}$ & $I-.63 Q-.39 U-.68 \mathrm{~V}$ & $I+.16 Q+.87 U-.46 \mathrm{~V}$ & $I-.16 Q-.87 U+.46 \mathrm{~V}$ \\
\hline $67.5^{\circ}$ & $I+.60-.39 U+.70 \mathrm{~V}$ & $I-.60 Q+.39 U-.70 \mathrm{~V}$ & $I+.10 Q-.87 U-.48 \mathrm{~V}$ & $I-.10 Q+.87 U+.48 \mathrm{~V}$ \\
\hline $90^{\circ}$ & $I+Q$ & $I-Q$ & $I+Q$ & $I-Q$ \\
\hline $112.5^{\circ}$ & $I+.63 Q+.39 U-.68 \mathrm{~V}$ & $I-.63 Q-.39 U+.68 \mathrm{~V}$ & $I+.16 Q+.87 U+.46 \mathrm{~V}$ & $I-.16 Q-.87 U-.46 \mathrm{~V}$ \\
\hline $157.5^{\circ}$ & $I+.60 Q-.39 U-.70 \mathrm{~V}$ & $I-.6 Q+.39 U+.70 \mathrm{~V}$ & $I+.10 Q-.87 U+.48 \mathrm{~V}$ & $I-.10 Q+.87 U-.48 \mathrm{~V}$ \\
\hline
\end{tabular}




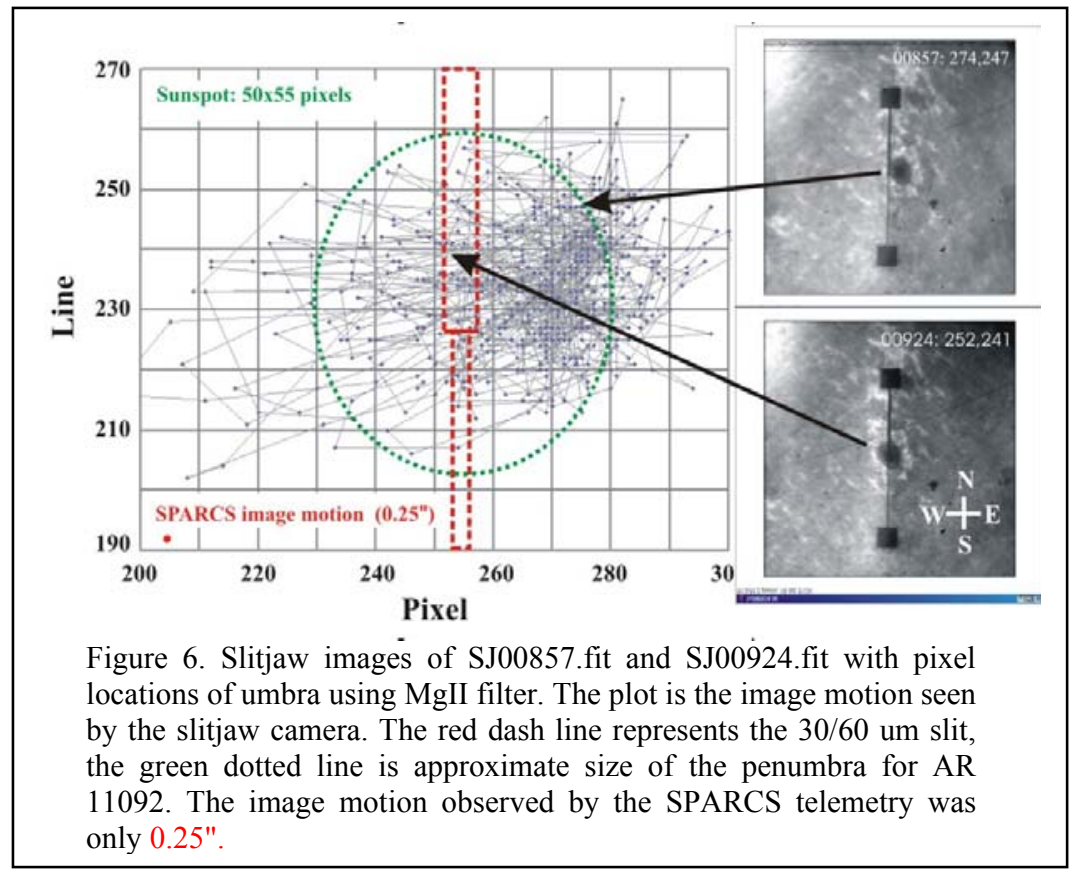

commands to eliminate this problem in future flights.
During the launch, the waveplate was suppose to start observations at $100 \mathrm{~km}$. Unfortunately, the waveplate bound up during this phase creating a timing problem between the data system and the waveplate controller which was not observed in our ground-based testing. With this timing error, the waveplate failed to rotate limiting SUMI to just linear polarization measurements $\left(\mathrm{FA}=0^{\circ}\right.$, blue in Table 2). As a result, there are no CIV circular polarization measurements.

After SUMI was returned from WSMR, extensive testing of the waveplate was done to determine the source for this failure. The failure was determined to be a timing error between the command to move and a polling request command. While a one microsecond delay in these commands was shown to resolve this failure, a one millisecond delay has been added to all of the microcontroller

\subsection{Pointing system problems}

During the flight, a pointing problem was not obvious in the realtime images from our slitjaw camera. Only after the flight when a movie was made of the slitjaw images was the problem obvious.

Figure 6 shows the random nature of the image motion. The telemetry (roll, pitch and yaw) from the SPARCS pointing indicated that the image motion was less than 0.5 " while the image motion in the SUMI slitjaw camera was two orders of magnitude larger. Analysis of the slitjaw motion and the changes in the gas pressure that controls the pointing indicated a direct relationship between the two. Since the slit did not move in the slitjaw images, that left the SUMI telescope or some "unknown" anomaly in the SPARCs pointing system. After a careful examination of the SPARC's data, the SUMI telescope became the leading candidate for the image motion.

While a failure in the epoxy joints holding the primary mirror to its holder might explain the image motion, the primary mirror holder had no tilt or alignment adjustments. All of the adjustments for the SUMI telescope are on the secondary holder. No problems were noted during the inspection of the epoxy joints on both the primary and secondary mirrors. Only after disassembling the secondary spider and checking the secondary tilt mechanism did the source for SUMI's pointing problem become obvious. Although the tilt mechanism was okay, two set screws on the focus mechanism were missing which allowed it to back away from the secondary tilt assembly. Without this support, the secondary was allowed to tilt $\pm 0.05^{\prime}( \pm 1.2$ arc minute in Figure 6).

While the image motion allowed SUMI to observe various structures in AR 11092, a scientific objective for this first mission is to measure the Stokes polarization from a simple active region. Therefore, stable pointing is required to integrate up enough photoelectrons to get the signal to noise $(\mathrm{S} / \mathrm{N})$ required to detect the polarizations levels that were expected in the Sun's transition region (Figure 14), especially when limited to linear polarization measurements. The consequences of this image motion will be described in greater detail in next section.

\section{DATA ANALYSIS}

One of the scientific goals of SUMI is to obtain the full Stokes polarization [I,Q,U,V] measurements on the MgII emission lines. AR 11092 would have been an ideal candidate for this first exploratory flight if the launch could have been delayed 3 days since the active region was a large simple H-type sunspot that would provide a "potential" magnetic field and allow us to compare photospheric magnetic field measurements with SUMI's chromospheric/transition region 


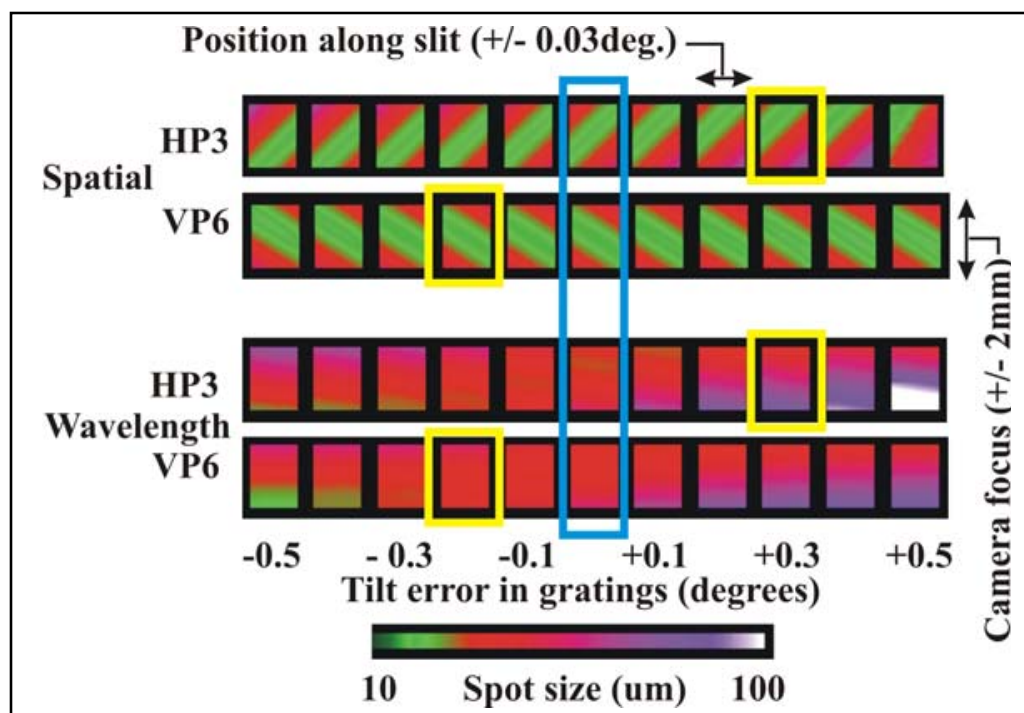

Figure 7. Zemax optical model showing wavelength and spatial resolution spot sizes across field of view of SUMI spectrograph cameras. measurements. With the failure of the waveplate, SUMI's data was limited to MgII linear polarization measurements (§2.1).

With this simple region (Figure 5), solar activity would be low and intensity crosstalk would not have been a problem. Therefore, each polarization channel (VP6 and HP3) could be analyzed independently. Completing the polarization analysis for each channel (VP6 and HP3, see Table 2), the software to correct for the instrumental errors between the two gratings would then be developed. Unfortunately, the waveplate failure limited our ability to calibrate/correct for instrumental errors between the two polarization channels. The expected instrumental errors are: (1) differences in the optical efficiency between the two gratings, (2) the X/Y magnification due to the different distances between the slit and the gratings, (3) small rotation errors in the images (CCD cameras and gratings) and (4) the aberrations over the field of view for the two gratings. Of those, the variation of the aberrations/spot size over the field of view of the VP6 and HP3 polarization channels is the most difficult error to correct. While a Zemax optical model can produce an "ideal" model for the differences between the two optical paths (Figure 7), the task becomes labor intensive when manufacturing (gratings: rulings, ruling axis versus toroidal axis, toroidal radii and stress induce mounting) and alignment (gratings, fold mirrors, cameras: optical axis position, tilt, and rotation) errors must also be considered. In Figure 7 the blue box shows the ideal alignment of the gratings but the actual alignment of the gratings during the SUMI observations depends on the mechanical structure maintaining the preflight calibration. The yellow boxes represent a possible misalignment of less than $\pm 0.3^{\circ}$ but subtracting those images could produce large instrumental signals.

\subsection{Requirements to obtain $10^{-3}$ linear polarization}

With a region so close to the limb, the linear polarization measurements are expected to be stronger. Unfortunately, the pointing system became the limiting factor in the MgII polarization analysis. In order to improve the signal to noise $(\mathrm{S} / \mathrm{N})$ of the polarization measurements, our earlier radiometry calculation indicated that eight (8) images would be required to get a $\mathrm{S} / \mathrm{N}$ of $\mathbf{4 0 4 8}$ for plage regions and $\mathbf{9 3 2}$ for quiet regions to meet our scientific goals. Eight images is not a large number but it assumes stable pointing (\$2.2) and exposures optimize for $80 \%$ of the well capacity for the MgII CCDs. Since the exposure times cannot change during the flight, the number of images must be different for plage and quiet regions that are centered onto the slit. Therefore, our post flight goal for the MgII linear polarization measurement is to obtain a minimum of 16 images that can be co-aligned to achieve our $\mathrm{S} / \mathrm{N}$ requirements.

With the uncertainties in our radiometry calculations, two exposure times were used for the MgII observations: 10ms and $100 \mathrm{~ms}$. Both of these exposure times were acceptable for the $30 \mu \mathrm{m}$ slit but when plage was observed in the $60 \mu \mathrm{m}$ slit, the $100 \mathrm{~ms}$ exposures were saturated. Therefore, most of our analysis has been limited to the $10 \mathrm{~ms}$ exposures.

In order to obtain enough images for our polarization analysis, a 5x144 "super-pixel" structure was developed and all of the MgII images with the sunspot in that super-pixel were sorted into a spreadsheet. Figure 8 shows the distribution of the MgII images within that super pixel grid. Comparing Figures 6 and 8, the slit is west of the sunspot in most of SUMI's images (the slit is in super pixel 8, black line in Figure 8). The next section will describe the calibration and alignment of the $10 \mathrm{~ms}$ MgII images that will be used to produce SUMI's linear polarization measurements. 


\subsection{MgII measurements}

SUMI obtain over $500 \mathrm{MgII}$ spectra of AR11092 during its flight. The maximum number of spectra with a $10 \mathrm{~ms}$ exposure time was in super pixel 13 which had 57 data sets (Figure 8). Therefore, it is that data set that has been used in our initial polarization analysis. While the slitjaw images "suggest" that SUMI has enough data sets to achieve our polarization resolution goal ( $>16$ images and $<10^{-3}$ ), the fine structure of the MgII emission requires additional processing to minimize the image motion effects in this data set. Figure 9 shows three data sets that were placed in super pixel 13 and the image motion along the slit.

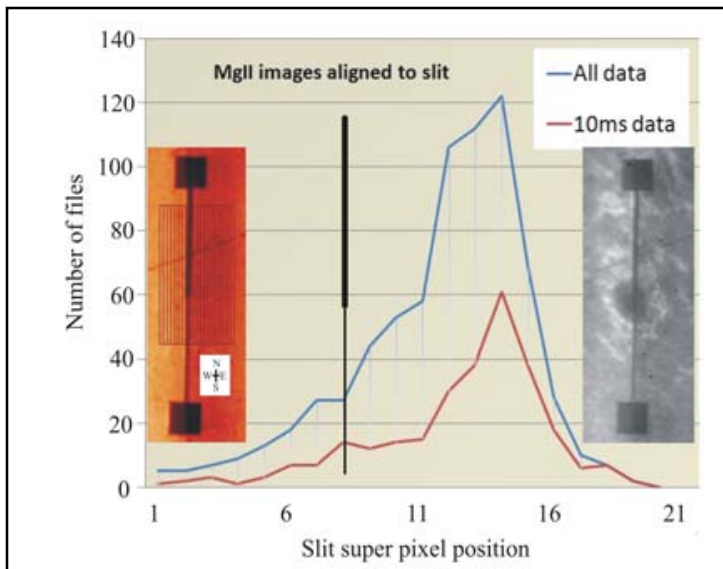

Figure 8. Distribution of MgII images in 5 pixel bins parallel to the slit. Bin 8 is center of sunspot on slit (right image, SJ00723 x/y position 245,230). In SUMI's observations, the sunspot is 20 to $30 \mathrm{SJ}$ pixels (10"-20") east of the slit.

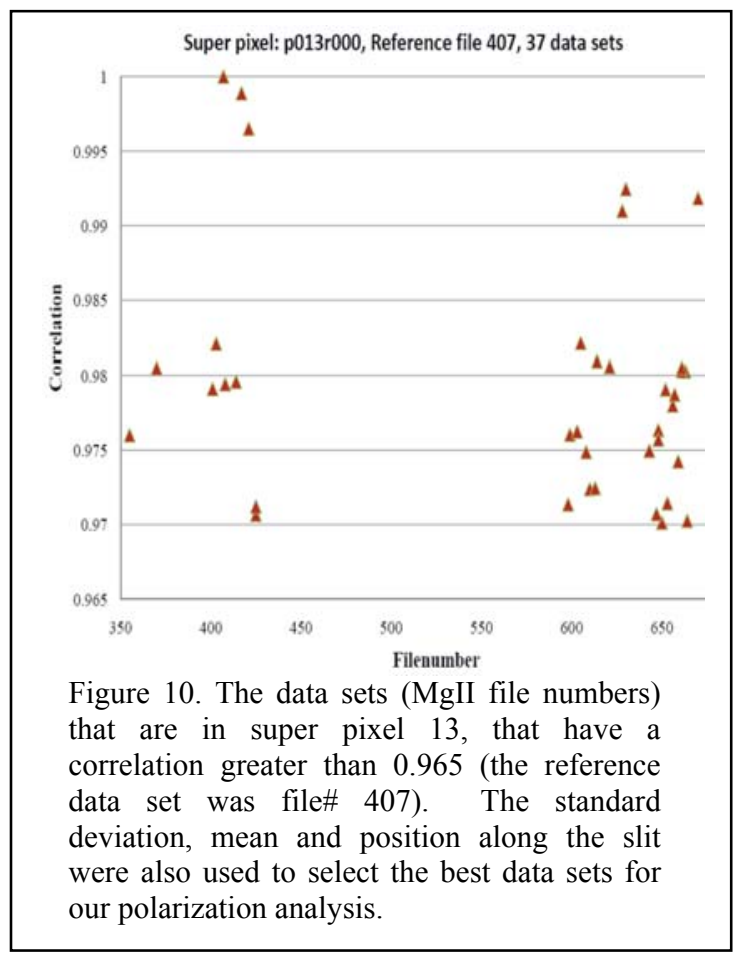

While slit jaw images and MgII spectra may be close in time, the effects of image motion appear to be larger in the spectra due to the limited field of view of the slit. Therefore an additional step in the MgII analysis is to minimize the image motion effects by doing a correlation analysis of all of the files in a super pixel. The yellow box in Figure 9 (file\# 417) is the MgII feature that was used to further reduce our data set. The data sets that will be used in our polarization analysis are shown in Figure 10.

In Figure 10, there are two distinct data sets. The first data set is early in the flight when there was a lot of image motion (files 350-430) and the second data set when the pointing and control system were becoming "stable" (files 580-670). Therefore, our analysis will use the second data set in our MgII polarization analysis.

Figure 11 shows the same data set as Figure 10 but has the data sorted with the movement along the slit (Y pixel position -250). Of the 37 data sets that met our correlation requirements (Figure 10), we are left with 26 data sets. 


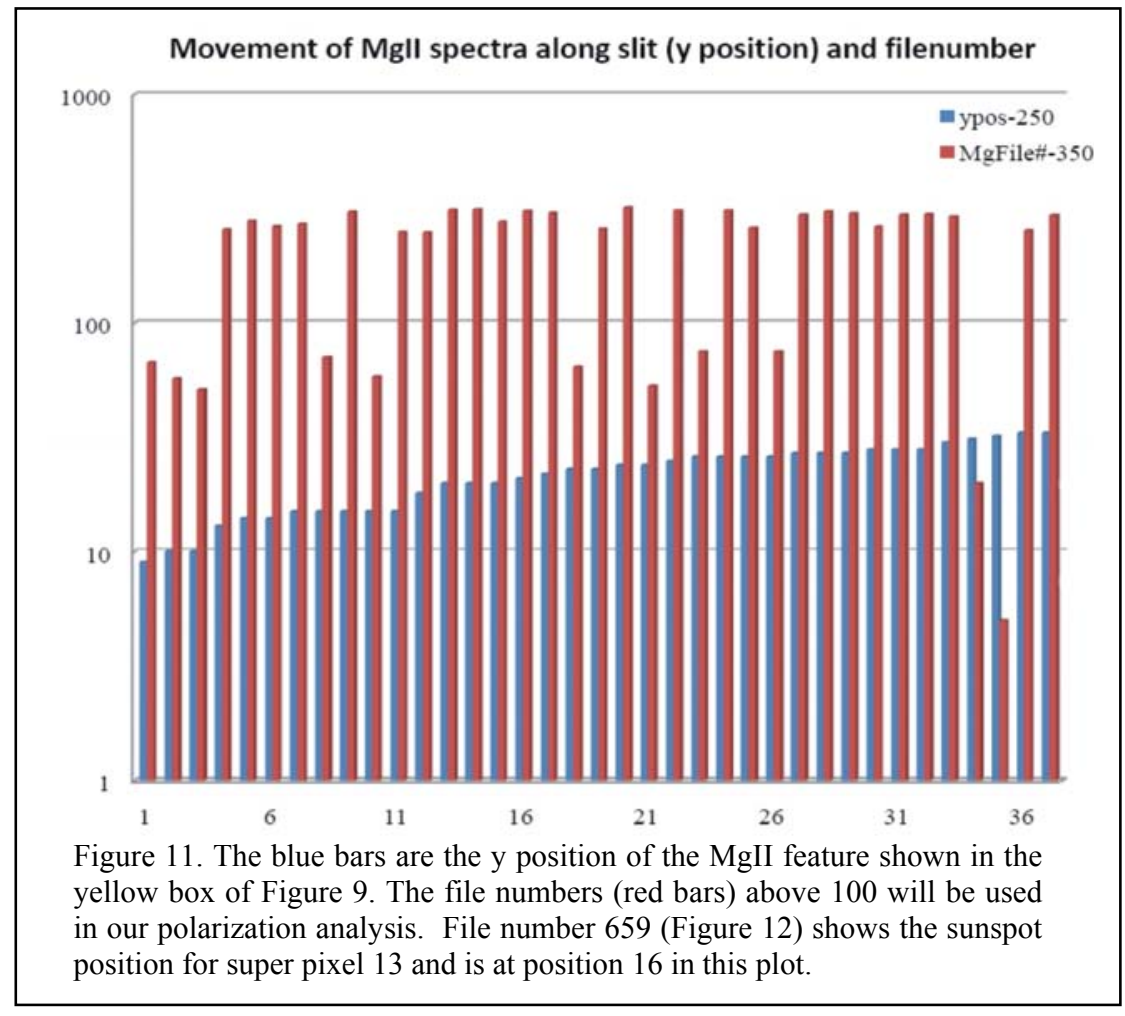

\subsection{Optimizing the MgII data}

While super pixel 13 has the most data sets, and hence the best chance for measuring the linear polarization in MgII, the location of the active region on SUMI's slit is not ideal. Figure 12 shows the MgII spectrum (file\# 659) for super pixel 13 and the corresponding MgII slitjaw image. Figure 13 shows the HMI photospheric measurements of the magnetic field. The HMI data was rotated to match the SUMI slitjaw images and the orientation of HMI's linear polarization measurements to match SUMI's analyzer. Since this region is nearly "potential", the magnetic structure that is observed in the photosphere should be "similar" to that observed at the MgII height.

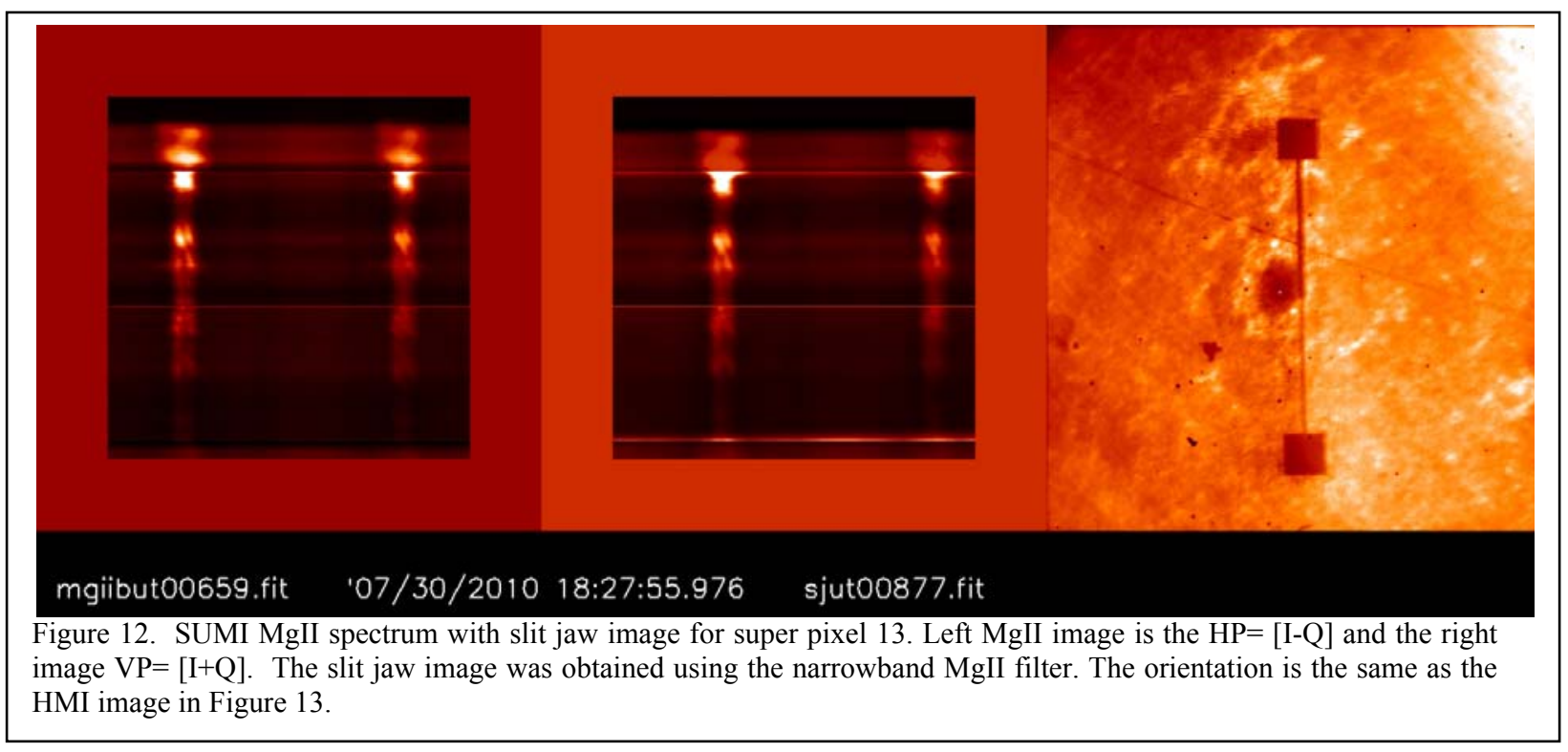




\subsection{MgII polarization analysis}

While we have identified the necessary number of data sets that can be processed to achieve our $10^{-3}$ polarization resolution, most of those data sets are late in the mission when the pointing system/secondary had "stabilized". At that time our slit, unfortunately, ended up on the west side of the active region where linear polarization is expected to be weak (Figure 15). Therefore, reducing the instrumental errors to $<10^{-4}$ will be required.

Some of the instrumental errors are not difficult to correct. The difference in the optical efficiencies of the two beams (HP3 and VP6) which includes the difference in the polarization reflectivity of the gratings ${ }^{13}$ and the electronic gain of the two cameras is a simple correction. There are two magnification corrections that must be made. In order to minimize the mechanical contact between the gratings during launch, the gratings are offset in the z-axis. Therefore, HP3 is closer to the telescope focus and has a slightly larger magnification at the detector than VP6. Since the gratings are toroidal, this magnification is different in the $\mathrm{X}$ and $\mathrm{Y}$ axes. To transform the HP3 images to the VP6 scale we must multiply the HP3 array size by $0.9578( \pm 0.0037)$ in the $\mathrm{X}$ axis and $0.9672( \pm 0.0032)$ in the $\mathrm{Y}$ axis.

For the MgII images, image rotation can be caused by the gratings and the cameras. While the source of this error is not important, determining this rotation angle has been difficult and will require looking at data not include in our super pixel data sets. Our current estimate for the rotation between the VP6 and HP3 images is $-0.539 \pm 0.442$. While the error "looks" small the uncertainty is large. The MgII lines are near the edge of images where this "small" rotation could be as large as 5 pixels. A program is being developed to automate the analysis of this error and will include all of our $10 \mathrm{~ms}$ images. We hope this will reduce the large uncertainty in the rotation angle.

The resolution difference between the two optical paths has not been quantified. While our Zemax model can show the resolution errors, it assumes that the grating alignment survived the launch (and landing). After the magnification and rotation errors have been corrected, the current approach will be to analyze the VP6 and HP3 independently as the image of a superpixel data set moves along the slit (Figure 11). Assuming that the image motion has been eliminated (wavelength axis), a Zemax model can be developed to match the observed change in resolution. If successful, this final correction might lead to a real solar linear polarization measurement.

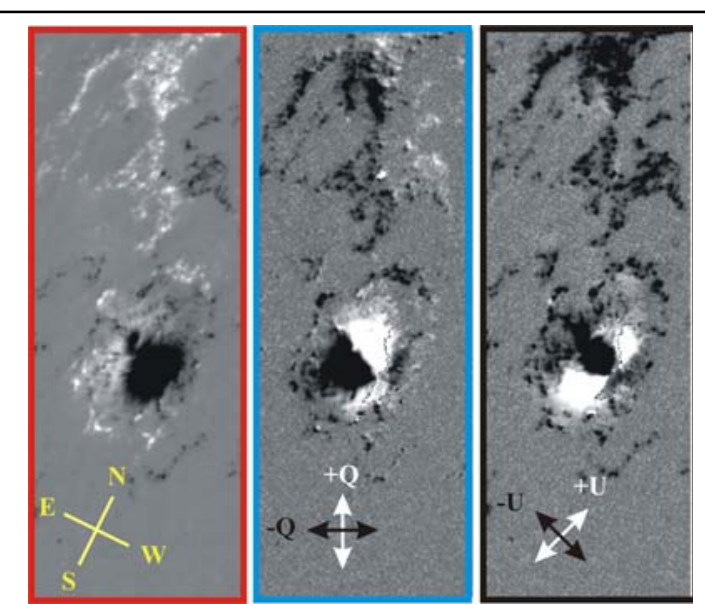

Figure 13. Stokes photospheric polarization maps obtain from the HMI vector magnetic field measurements on the Solar Dynamics Observatory observed on 7/30/2011 at 1800UT. The polarization images (V/I left image/red box and $\mathrm{Q} / \mathrm{I}$ center/blue box) images were rotated to match the SUMI slit jaw images and the HMI linear polarizations ( $\mathrm{Q}$ and $\mathrm{U}$ ) were rotated to the same coordinate system as the SUMI analyzer $(+/-\mathrm{Q}=\mathrm{VP}-\mathrm{HP})$. The only transition region polarization measurement made by SUMI is the $\mathrm{Q}$ polarization (see \$2.1). The E/W orientation is not the same as Figure 6 and 8.

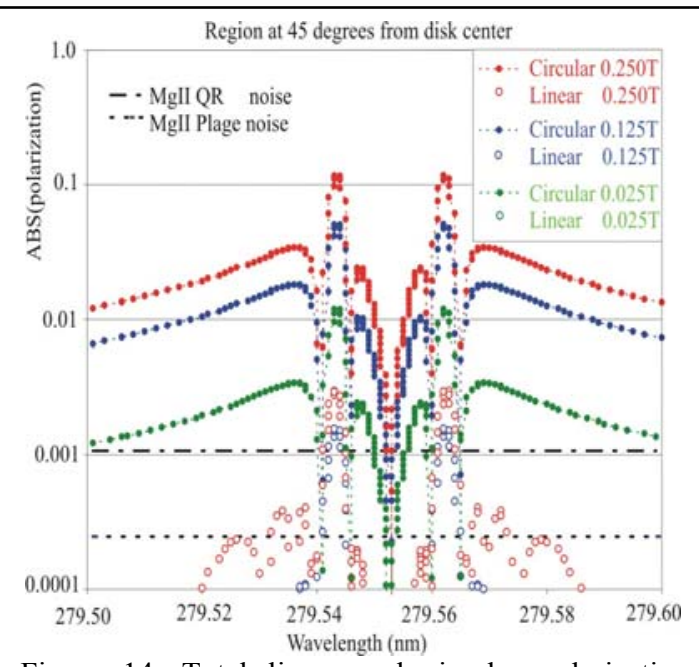

Figure 14. Total linear and circular polarization expected for magnetic fields $\left(\mathrm{T}=10^{4} \mathrm{G}\right)$ that vary with wavelength $(\mathrm{nm})$. The expected noise levels for three magnetic field strengths for the MgII measurements are indicated by horizontal dash lines. The radiative transfer model was developed by Dr. Han Uitenbrock/NSO for SUMI (private communication). 


\section{SUMMARY}

The failure of the waveplate limited us to only MgII linear polarization measurements. While the active region that we observed on 7/30/2010 did not meet our launch criteria (within one half the solar diameter) for full Stokes polarization measurements, the region would have been ideal for linear polarization measurements. Unfortunately, the image motion and the "final" position of the slit has made this linear polarization analysis difficult.

Although the probability of reducing SUMI's instrumental errors below our goal of $10^{-4}$ and obtaining a "real" solar MgII linear polarization signal is low for this initial engineering flight, we will continue to develop our scientific software since this research will be important in the data analysis of SUMI's next flight which is scheduled for the summer of

Figure 15. SUMI's slit overlaid onto an
AIA 17.1nm image taken on 7/30/2010.
The AIA image is rotated to match
SUMI's slit jaw images. Most of SUMI's
data is in the quiet region west of AR
11092.
2012.

\section{REFERENCES}

[1] Gary, G. A., "Plasma Beta above a Solar Active Region: Rethinking the Paradigm," Solar Phys., 203, 71 (2001).

[2] Rosner, R., Low, B. C., and Holzer, T. E., "Physical processes in the solar corona," [Physics of the sun], D. Reidel Publishing Co, 135-180 (1986).

[3] Moore, Davis, and Hathaway, "High-Resolution Solar Magnetography from Space: Beyond Solar-B," http://solarscience.msfc.nasa.gov/Beyond_Solar-B.shtml, (2001)

[4] Davis, J., West, E., Moore, R., Gary, G., Kobayashi, K., Oberright, J., Evans, D., Wood, H., Saba, J. L. R., and Alexander, D., "MTRAP: The Magnetic Transition Region Probe," Proc. SPIE 5901, 273-280 (2005).

[5] Gurman, J. B., "The MG II H line in sunspot umbrae," Solar Phys., 90, 13-15 (1984).

[6] Uitenbroek, H., "Multilevel Radiative Transfer with Partial Frequency Redistribution," Astrophysical Journal, 557, 389-398 (2001).

[7] Peter, H., "On the nature of the transition region from the chromosphere to the corona of the Sun," A\&A, 374, $1108-1120$ (2001).

[8] West, E. A., Porter, J. G., Davis, J. M., Gary, G. A., Rabin, D. M., Thomas, R. J., and Davila, J. M., “Overview of the Solar Ultraviolet Magnetograph Investigation," Proc. SPIE 4139, 350-361 (2000).

[9] West, E. A., Porter, J. G., Davis, J. M., Gary, G. A. and Adams, M., "Development of a polarimeter for magnetic field measurements in the ultraviolet," Proc. SPIE 4481, 109-117 (2001).

[10] West, E. A., Kobayashi, K., Davis, J. M., and Gary, G. A., "The Solar Ultraviolet Magnetograph Investigation Sounding Rocket Program," Proc. SPIE 6689, 66890E-12 (2007).

[11] Kobayashi, K., West, E. A. and Noble, M., "Polarization measurements in the Vacuum Ultraviolet," Proc. SPIE 5888, 1-12 (2005).

[12] West, E. A., Porter, J. G., Davis, J. M., Gary, G. A., Kobayashi, K. and Noble, M., "The Solar Ultraviolet Magnetograph Investigation: Polarization Properties," Proc. SPIE 5901, 226-235 (2005).

[13] Kobayashi, K., West, E. A., Davis, J. M. and Gary, G. A., "Polarization measurements on SUMI's TVLS gratings", Proc. SPIE 6682, 1-12 (2007).

[14] Thomas, R. J., “Toroidal varied line-space (TVLS) gratings, ” Proc. SPIE 4853, 411-418 (2003).

[15] Poletto, L. and Thomas, R. J., "Stigmatic Spectrometers for Extended Sources: Design with Toroidal Varied Line-Space (TVLS) Gratings, ” Applied Optics, 43, 2029-2038 (2004). 